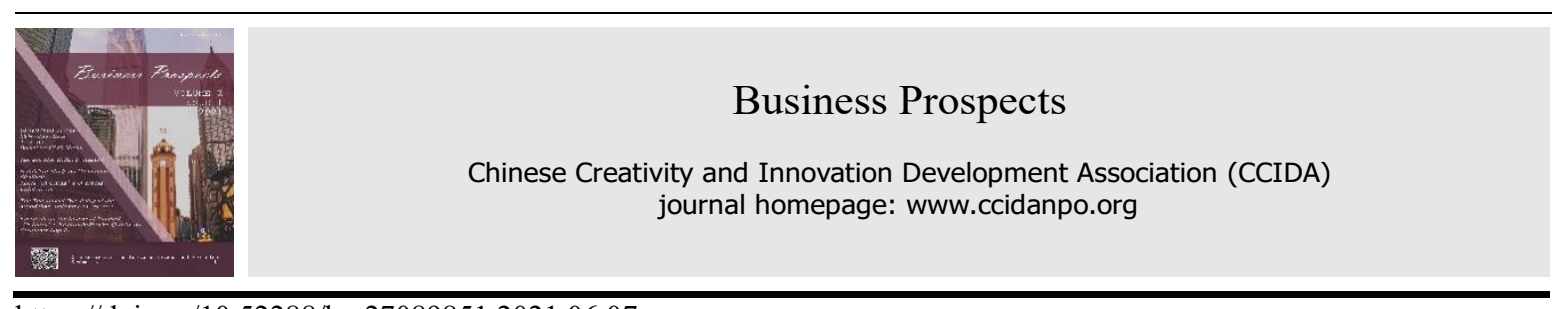

https://doi.org/10.52288/bp.27089851.2021.06.07

\title{
Analysis and Research on the Effect of Online Celebrities Carrying Goods in Live Broadcast
}

\author{
Gong-Jian Zhou ${ }^{1, *}$ \\ ${ }^{1}$ Associate Professor of School of Management, Xiamen University Tan Kah Kee College \\ * Correspondence: 63223748@qq.com
}

Received: 2021.02.02; Accepted: 2021.03.15; Published: 2021.06.01

\begin{abstract}
Since 2020, live broadcasting with goods has become a hot topic, and the Internet's popular KOL (key opinion leader) has emerged in an endless stream. From the perspective of marketing and consumer behavior, this paper takes KOL as an example to explore the reasons for the success of Internet celebrity live broadcasting, and analyzes the current situation and pattern of live broadcasting, so as to provide reference and suggestions for enterprises and individuals who want to make profits through live broadcasting.
\end{abstract}

Keywords: Online Popularity; Live Broadcast with Goods; E-Commerce Platform; Fan Economy; Trilateral Market

\section{Analysis on The Cause of KOL Explosion}

In the business era of fierce competition, key opinion leader (KOL) emerges in endlessly. A well-known Internet celebrity anchor as a lipstick brother quickly occupied the attention of consumers, and always occupied the high topic degree of various Internet platforms. Behind his popularity, it is inseparable from the creation of differentiated IP, consumer psychology, platform and industrial chain advantages, cross-border marketing and fan economy (Zhong, 2020).

\subsection{Building Differentiated Super IP}

In 2015 , a KOL also an Internet celebrity who worked as a beauty consultant at the counter of L'Oreal, created a sales miracle, and were invited to share his experience in the headquarters of L'Oreal. In 2016, he took part in the Taobao live broadcast competition of "Ba networks red", successfully came out, signed a contract with MeiOne, and entered the field of beauty live broadcast.

In 2018, the anchor was awarded the title of "lipstick brother" by the outside world for successfully challenging the Guinness world record of "the most lipstick in 30 seconds" (Jiang, 2020). The head KOL's behavior, words and deeds, appearance has a distinct label. He uses unique sales techniques such as "oh my God", "all girls, buy it", "my God", "Mom, it's so beautiful" to constantly narrow the distance between himself and female consumers, increase customers' trusts and stimulate their desire to buy.

At the same time, his popularity reflects the contrast brought by gender conflict. The male spokesperson's promotion of women's products breaks the cognitive framework of female audience. The unique aesthetic of the male Internet celebrity anchor transcends gender barriers, brings high topic degree, and causes the pursuit of female consumers (Yang et al., 2020).

\subsection{Insight into Consumer Psychology}

Another essential element of KOL's success is to understand the psychology of consumers, focus on the needs of consumers, and provide the products that consumers need. Focusing on demand is mainly reflected in the aspects of quality control, low price, reducing decision-making cost, symbolic consumption and scenario construction.

In quality inspection team of the famous online anchor, all the members are graduate students from the 
fields of chemical testing and food research. The professionalism of the team ensures excellent quality control and further forms the effect of public praise. He and consumers have formed a bond of trust with high customer loyalty. One of the requirements of KOL team's cooperation with the brand sides is the lowest price in the whole network in an attempt to provide the best discount to consumers. Through the lowest promotion means in the whole network to stimulate consumers, the instant stimulation to price sensitive consumers is formed. KOL's direct recommendation reduces consumers' choices and decision-making costs, enables consumers to focus on brands, and make better purchase decisions.

In addition, he uses specific language to sell scripts, such as "one for each person, yellow skin must enter, Queen's color number" and so on, forming "symbolic consumption". Consumers increase their sense of selfidentity and group identity through purchase (Chen, 2020). In addition, the scene construction in sales discourse has a strong stimulation to the human brain. It can induce customers to recognize products through specific scenes, effectively touch consumers' pain points, arouse consumers' emotional resonance, and stimulate consumers' desire to buy. Continuous scene stimulation may lead to consumers' impulsive consumption, compulsive consumption, and addictive consumption under pressure situations.

\subsection{Platform Traffic Support and Industry Chain Driving Force}

In 2016, Taobao entered the e-commerce live broadcast, and this head KOL appeared in the public eye as "Taobao first brother". In 2018, Taobao's live broadcast went straight ahead, and the top KOL in the Internet celebrity anchor formed a monopoly. In 2020, the development of online network completely catalyzed the formation of live broadcast outlets which made live broadcast with goods the most popular new format.

Taobao live broadcasting, as a pioneer of live broadcasting e-commerce, has a mature process system and occupies a large market share. The two heads of Taobao anchor lineup, KOL, have successfully caught the wind of e-commerce live broadcasting. By using the network flow of Taobao live broadcasting, it has become the synonym of "professional delivery" in the anchor industry (Zhang, 2020).

In addition to the network flow of the platform, the team also uses the whole network content marketing to bring continuous network flow, forming a halo effect. Tiktok, Xiao Hong, micro-blog, WeChat and other App can see the content of KOL live broadcast on the head, and further enhance the understanding and familiarity with KOL.

\subsection{Cross Border Marketing and Fan Economy}

In the period of rapid development of online network in 2020, CCTV playwright Zhu Guangquan and Li Jiaqi will broadcast live with the theme of "thank you, fight for Hubei" to do public welfare for Hubei.

The cross-border marketing of the hosting and live broadcasting circles of "Xiao Zhu Peiqi" has attracted a lot of attention and flow. In addition to the cross-border marketing focusing on public welfare, this Internet celebrity anchor also makes good use of fan economy to enhance user immunity and increase revenue. From a popular female star to a modern flow Xiaosheng, from a comedian, a comedian to a literary star, each of the stars with his partner has its own characteristics. The event of cooperation with the stars makes the live broadcast room of this network popular hot search (Jiang, 2020). The content of his live broadcast has also been spread among the fans, which has increased the popularity of the live broadcast room in the fan circle. The fans' purchase, however, has further brought greater turnover of the live broadcast room.

\section{Analysis of Direct Air Outlet}

\subsection{Development Status of Live Broadcasting}

\subsubsection{Major Companies have Launched Live Broadcast Platforms}

Besides the three live broadcasting platforms, Kwai, Tiktok and Joule, many spells like Jingdong, Microblog, Suning, Xiaohongshu, and B stations also invest in developing live broadcast business. Taking Xiaohongshu as an example, in 2020, Xiaohongshu will issue a brand support plan to connect brands from the aspects of capital, flow and anchor with goods. It will provide preferential policies such as free entry, reduced commission and 10 billion flow support for businesses with the aim to follow the first-line live broadcasting platform and occupy the market share of live broadcasting. 


\subsubsection{The Anchor Group Is Expanding}

In addition to KOL, the head of the Internet celebrity anchor, traffic stars, hosts and entrepreneurs of large enterprises with good reputation, which are not Internet celebrity groups with their own traffic, also carry out live selling, bring traffic and high topic degree to the enterprise, and further create a lot of profits for the enterprise. For example, a well-known actress's first show attracted 21 million viewers and eventually reached 148 million turnovers (Yang et al., 2020).

\subsubsection{Various Live Broadcast Forms}

In addition to the common live broadcasting room, live broadcasting (stall, seafood market, orchard) and long-term daily broadcasting in shopping malls and shops have been developed (Zhang, 2020). For example, Netease Yanxuan has set up a live broadcast e-commerce base in Guizhou, which grafts the bridge between traditional businesses and Internet celebrity anchor in the way of live broadcast, promotes the improvement and development of industrial supply chain in Qianxinan Prefecture, helps local agricultural products merchants sell successfully, and promotes the process of poverty alleviation in Qianxinan Prefecture.

\subsection{Reasons for the Success of Head KOL-Trilateral Market Effect}

The three sides of the trilateral market effect correspond to suppliers, consumers and platforms respectively. In the face of suppliers, platforms and consumers, head KOL have a strong voice. First of all, for suppliers, the head anchor is to complete the KPI and achieve the sales guarantee, but the head KOL has a strong bargaining power in terms of pit fee and product discount.

For consumers, head KOL is the guarantee of product quality and low price. KOL has successfully established a bond of trust with consumers and formed word-of-mouth marketing in the circle of consumers. For the platform, the head KOL is the guarantee of flow. For example, on Taobao live broadcasting platform, the four head KOLs with distinctive characteristics are Li Jiaqi, Wei Ya, Wang Han and Liu Tao, which attract a large number of "post-90s" and "post-00s" customer groups, and implant the brand proposition of "professional live shopping on Taobao live broadcasting" that Taobao live broadcasting wants to convey into consumers' minds. In the hearts of consumers, Taobao live professional impression, in the number of good reputations at the same time, attract more customers (Zhang, 2020).

Therefore, the head KOL has formed a virtuous circle with suppliers, consumers and platforms, achieving the trilateral market effect, which is also the reason for the success of the head KOL under the direct air outlet.

\section{Discussion and Suggestions on The Possibility of Successfully Carrying Goods under the Direct Air Outlet}

First of all, in the case of strong bargaining power of KOL, suppliers of major brands need to consider with KOL team to better integrate the tonality of brands into the live broadcast situation in addition to cooperating with KOL to achieve sales targets. In the situational scenario, through the analysis of sales data and purchase volume, we can get insight into the psychology of consumers, adjust the tonality of products that do not meet the consumer demand, guide KOL to construct the situation, make consumers more aware of the brand, and form the benign growth of the brand.

Secondly, the enterprises can combine their own advantages and values, and embed the live broadcast into the inherent channel advantages with attention to the way of marketing and publicity. For example, if the luxury industry wants to develop live broadcasting, it cannot focus on the low price of traditional live broadcasting. The brand tonality will be destroyed and the luxury brands will lose its competitive advantage.

Finally, live broadcasting has become a new outlet. However, due to the rapid spread of information and the rapid response of large platforms, flow has been monopolized by head anchors and stars. Without platform support, ordinary people have few opportunities and high cost to enter the live broadcasting industry. Entering the live broadcasting platform requires registering the company, doing user visits, finding out the selling points of the products, recording user data, and doing live broadcasting research. Careful investment is the best strategy for those who plan to enter the live broadcasting platform in the future.

\section{Conclusion}

At present, e-commerce live broadcasting has reached the outlet. The live broadcasting with goods has gradually formed a stable pattern and the mode of "platform traffic + stars' own traffic" has accelerated the 
formation of this pattern. Flow and resources tend to be concentrated in KOL. If ordinary people want to bring goods through live broadcast, it is difficult to gather enough flow in a short period. Enterprises cooperating with KOL can combine their own advantages and values to guide KOL to construct a situation so consumers can perceive the brand more and finally form a healthy growth of the brand.

\section{References}

1. Chen, R. (2020). Analysis of Language Features and Content Points of E-commerce Anchor Li Jiaqi. Journal of News Research, 11(12): 56-57.

2. Jiang, H. F. (2020). Analysis of the Current Development of Live E-commerce. China Circulation Economy, 22: 7-9.

3. Yang, R., Chen, Y. H., \& Wang, Y. (2020). Analysis on the Value Realization Path of Internet Celebrity Live Broadcast with Goods. New Media Research, 5: 56-57.

4. Zhong, T. (2020). Analysis on Development Factors, Driving Force and Growth Sustainability of Live Broadcast ECommerce. Commercial Economy Research, 10(18): 85-88.

5. Zhang, Y. M. (2020). Online Live Broadcast with Goods Dissemination Strategy-Take Blogger Li Jiaqi as an Example. Southeast Communication, 4: 88-91.

(Editors: Yilia Zhang \& Amy Li) 